\title{
Qualidade das juntas coladas de madeira de cinco espécies de Eucalyptus com adesivos acetato de polivinila e resorcina-formaldeído
}

\section{Quality of wood bonded joints of five species of Eucalyptus with polyvinyl acetate and resorcinol-formaldehyde adhesives}

\author{
Rosilani Trianoskil', Setsuo Iwakiri|* e Ghislaine Miranda Bonduellel
}

\begin{abstract}
1 Universidade Federal do Paraná. Departamento de Engenharia e Tecnologia Florestal. rosillani@ gmail.com; ghislainebonduellemiranda@gmail.com * Autor correspondente. setsuo.ufpr@gmail.com
\end{abstract}

\section{RESUMO}

O objetivo deste trabalho foi avaliar o potencial das madeiras de Eucalyptus benthamii, Eucalyptus dunnii, Eucalyptus grandis, Eucalyptus saligna e Eucalyptus urophylla, para produção de painéis colados lateralmente (EGP) e madeira laminada colada (MLC). As propriedades físicas e químicas das madeiras foram determinadas com base nas normas COPANT 461/459:1972 e TAPPI 204/252:2002. Foram confeccionadas juntas coladas com adesivo acetato de polivinila (PVA), com gramaturas de $150 \mathrm{~g} \mathrm{~m}^{-2} \mathrm{e} 200 \mathrm{~g} \mathrm{~m}^{-2}$, e resorcina-formaldeído (RF), com $200 \mathrm{~g} \mathrm{~m}^{-2}$ e $300 \mathrm{~g} \mathrm{~m}^{-2}$. A qualidade de colagem foi avaliada por meio de ensaios de cisalhamento, com base na norma EN 13354:2008. Todas as espécies apresentaram densidade básica de média a baixa e anisotropia de contração de média a alta. O teor de extrativos totais e pH estão dentro da faixa referenciada na literatura. As avaliações dos resultados com base nos requisitos da norma EN 15353:2008 para $5^{\circ}$ percentil inferior indicaram que as espécies E. benthamii, E. dunnii, E. grandis e E. saligna, apresentam potencial para produção de painéis EGP com adesivo PVA, para o uso interno, e externo com cobertura. Para a colagem com adesivo RF, o E. dunni apresentou melhores resultados, sendo indicativo do seu bom potencial para a produção de madeira laminada colada, visando aplicações estruturais em ambientes interno úmido e externo. Para os dois tipos de adesivos o aumento na gramatura não afetou os resultados de cisalhamento das juntas coladas.

PALAVRAS CHAVE: anisotropia, cisalhamento da linha de cola, extrativos, falhas na madeira, madeira laminada colada, painéis EGP.

\section{ABSTRACT}

The objective of this work was to evaluate the potential of wood of Eucalyptus benthamii, Eucalyptus dunnii, Eucalyptus grandis, Eucalyptus saligna and Eucalyptus urophylla for the production of edge glued panels (EGP) and glued laminated timber (Glulam). Physical and chemical properties of the wood were determined based on the COPAN 461/459:1972 and TAPPI 204/252:2002 standards. Glued joints were manufactured in resin weights of $150 \mathrm{~g} \mathrm{~m}^{-2}$ and $200 \mathrm{~g} \mathrm{~m}^{-2}$ for polyvinyl acetate (PVA) and $200 \mathrm{~g} \mathrm{~m}^{-2}$ and $300 \mathrm{~g} \mathrm{~m}^{-2}$ for resorcinolformaldehyde $(\mathrm{RF})$. Bonding quality was evaluated by shear tests based on EN 13354-2008 standard document. All species presented medium to low basic density and medium to high contraction anisotropy. The total extractives content and $\mathrm{pH}$ are within the range referenced in the literature. The evaluation of the results based on the requirements of standard document EN 15353-2008 for the 5th lower percentile indicated that the E. benthamii, E. dunnii, E. grandis and E. saligna species showed high potential for the production of EGP with PVA adhesive, for indoor and outdoor use with coverage. For RF adhesive bonding, E. dunni showed better results, being indicative of its good potential for the production of glued laminated timber - GLULAM, for structural applications in the external environment. For both types of adhesives, the increase in weight did not affect the shear results of bonded joints.

KEYWORDS: anisotropy, glue line shear test, extractives, wood failures, glued laminated wood, edge glued panel. 


\section{INTRODUÇÃO}

Os plantios das espécies do gênero Eucalyptus no Brasil compreendem uma área aproximada de 5,6 milhões de hectares, sendo que, as espécies como E. grandis, E. saligna e E. dunnii, já têm sido amplamente empregadas comercialmente para produção de celulose e papel, carvão vegetal, painéis aglomerados e de fibras de madeira, e, em menor escala, para laminados, compensados e madeira serrada. Dentre as espécies menos utilizadas para plantios comerciais, como Eucalytpus urophylla e Eucalyptus benthamii, elas merecem atenção especial em função do seu potencial silvicultural e boas características de sua madeira. O Eucalyptus urophylla é uma espécie que apresenta grande potencial de crescimento em termos de plantios, devido à boa produtividade e qualidade da sua madeira. A espécie urophylla apresenta densidade básica média de 0,56; com possibilidades de utilização para os mais diversos fins, como celulose e papel, chapas duras, serrarias e carvão (Scanavaca Jr y Garcia, 2004). Já o Eucalyptus benthamii, é uma espécie com densidade básica média de 0,47; apresenta textura fina a média, grã irregular, e seu uso vem sendo estudada para diversas finalidades, como pallets, celulose, energia e compensados (Martins, 2011).

A utilização da madeira na forma de serrados apresenta limitações como dimensões, anisotropia e defeitos naturais que afetam as suas propriedades físicas e mecânicas. Entretanto, por meio da colagem de madeiras com emprego de adesivos é possível obter produtos reconstituídos de madeira, com maiores dimensões, maior estabilidade dimensional e melhor distribuição da resistência mecânica, com ganhos em qualidade e relação custo-benefício (Iwakiri, 2005).

Dentre os produtos reconstituídos de madeira, encontram-se os painéis colados lateralmente (EGP - Edge glued panel) e madeira laminada colada (MLC - madeira laminada colada, GLULAM - glued laminated wood). O EGP é um painel composto por sarrafos colados lateralmente, os quais podem ser unidos por emendas de topo "finger joints" (Tienne, Nascimento, Garcia e Silva, 2011). Sua produção permite a utilização de peças estreitas e curtas, com defeitos pequenos ou leves, resíduos de serrarias e de outras indústrias de base florestal, aumentando assim o rendimento por meio do reaproveitamento de um material que era descartado ou queimado como biomassa para geração de energia (Danawade, Malari, Patil e Hanamapure, 2014). Para a produção de painéis colados lateralmente, os adesivos à base de acetato de polivinila (PVA) são os mais utilizados (Lopes et al., 2013). O PVA é um adesivo resultante da polimerização do acetato de polivinila, sendo popularmente conhecido como "cola branca", e muito utilizado pela indústria da madeira e móveis para uso interior. O produto colado com PVA apresenta alta resistência mecânica em ambiente seco, porém limitações de uso em ambientes com altas temperaturas e umidade relativa (Pizzi, 1983).

Já, a madeira laminada colada, é uma peça estrutural constituída de tábuas com espessura variando de $10 \mathrm{~mm}$ a $50 \mathrm{~mm}$, unidas por adesivo, com as fibras de todas as lâminas paralelas ao comprimento da peça. As lâminas (tábuas) podem ser de diferentes espécies de madeira, espessuras, larguras e comprimentos, que são coladas borda a borda, obtendo-se peças de dimensões e qualidade desejadas. $\mathrm{O}$ adesivo mais empregado na fabricação de MLC é a resorcina-formaldeído, devido à sua alta resistência ao calor e umidade (Marra, 1992). A colagem de duas peças de madeira depende de vários fatores como a sua estrutura anatômica, massa específica e porosidade. Iwakiri (2005) relatou que as propriedades anatômicas da madeira estão relacionadas diretamente com as ações de mobilidade do adesivo na formação de "ganchos" de ligação entre as faces de colagem. A porosidade tem uma relação inversa com a massa específica, ou seja, madeira com alta massa específica possui menos espaços vazios e, consequentemente, dificulta a penetração do adesivo, resultando na baixa ancoragem e adesão.

A quantidade de adesivo (gramatura) na formação da linha de cola entre duas peças de madeira é um dos parâmetros específicos para promover uma boa colagem. De acordo com Fonte y Trianoski (2015) quando a gramatura é menor, ou maior que a ideal, a resistência do produto colado pode ser alterada. Gramaturas muito baixas 
implicam em resistência inferior devido à aderência e ancoragem insuficientes. Já, no caso de gramaturas elevadas, pode ocorrer vazamento do adesivo em excesso pelas bordas dos painéis, resultando em prejuízos econômicos (Marra, 1992). Iwakiri et al. (2014) afirmaram que a faixa de gramaturas mais empregadas pelas indústrias varia de $180 \mathrm{~g} \mathrm{~m}^{-2}$ a $220 \mathrm{~g} \mathrm{~m}^{-2}$ para o PVA e de $350 \mathrm{~g} \mathrm{~m}^{-2} \mathrm{a}$ $400 \mathrm{~g} \mathrm{~m}^{-2}$ para RF.

\section{OBJETIVOS}

Tendo em vista a necessidade de estudos sobre a utilização de madeiras de eucalipto provenientes de plantios florestais, para obtenção de produtos de maior valor agregado, este trabalho teve como objetivo, avaliar o potencial de uso de Eucalyptus benthamii, Eucalyptus dunnii, Eucalyptus grandis, Eucalyptus saligna e Eucalyptus urophylla, para produção de painéis colados lateralmente e madeira laminada colada.

\section{MATERIAIS E MÉTODOS}

Foram utilizadas nesta pesquisa, madeiras das espécies de: Eucalyptus benthamii, com 13 anos de idade, proveniente de Palmeira - SC; Eucalyptus dunnii, Eucalyptus grandis e Eucalyptus saligna, com 7 anos, de Rosário - RS; e, Eucalyptus urophylla, com 16 anos, de Corupá - SC. Foram coletadas três árvores por espécie, as quais foram seccionadas em toras com 2,0 m de comprimento e desdobradas em tábuas com $25 \mathrm{~mm}$ de espessura. Após secagem em estufa de laboratório com circulação forçada de ar, ao teor de umidade médio de $12 \%$, as tábuas foram serradas e aplainadas em sarrafos com dimensões finais de $22 \mathrm{~mm}$ (espessura) $\times 55 \mathrm{~mm}$ (largura) $\times 310 \mathrm{~mm}$ (comprimento) .

Das tábuas centrais das toras (posição radial), foram retirados os corpos-de-prova para determinação de propriedades físicas (densidade básica e anisotropia de contração), com base nos procedimentos das normas técnicas COPANT 461:1972 (Comissão Panamericana de Normas Técnicas [Copant], 1972a) e COPANT 459:1972 (Copant, 1972b), e propriedades químicas (teor de extrativos totais e $\mathrm{pH}$ ), com base nas normas técnicas
TAPPI 2002:2002 (Technical Association of the Pulp and Paper Industry [Tappi], 2002a) e TAPPI 252:2002 (Tappi, 2002b).

Para a colagem de sarrafos, foram utilizados os adesivos a base de acetato de polivinila (PVA D4), com gramaturas de $150 \mathrm{~g} \mathrm{~m}^{-2}$ e $200 \mathrm{~g} \mathrm{~m}^{-2}$, e resorcinaformaldeído (RF), com gramatura de $200 \mathrm{~g} \mathrm{~m}^{-2}$ e $300 \mathrm{~g} \mathrm{~m}^{-2}$. O tempo de prensagem empregado foi de duas horas para o PVA e de cinco horas para a RF. O adesivo foi aplicado com pincel sobre uma das faces e os sarrafos foram colados aos pares, com a aplicação de pressão específica de 0,65 $\mathrm{MPa}$. O delineamento experimental consistiu em cinco espécies, dois adesivos e duas gramaturas, totalizando 20 tratamentos, conforme apresentado na tabela 1 .

Após a prensagem, as juntas coladas foram acondicionadas na câmara climática à temperatura de $20{ }^{\circ} \mathrm{C}$ $\pm 3{ }^{\circ} \mathrm{C}$ e umidade relativa de $65 \% \pm 5 \%$. Para as juntas coladas com PVA, foram retirados corpos de prova para ensaios de cisalhamento da linha de cola, nas condições a seco (após climatização), a úmido (após imersão em água à temperatura de $20{ }^{\circ} \mathrm{C}+3{ }^{\circ} \mathrm{C}$ por $24 \mathrm{~h}$ ) e fervura (após imersão em água quente à temperatura aproximada de 100 ${ }^{\circ} \mathrm{C}$ por $6 \mathrm{~h}$, e $1 \mathrm{~h}$ de resfriamento em água à $20^{\circ} \mathrm{C}+3^{\circ} \mathrm{C}$ ), conforme os procedimentos descritos na norma técnica EN 13354 (European Standards [EN], 2008). Para as juntas coladas com RF, foram retirados corpos de prova para ensaios de cisalhamento da linha de cola, nas mesmas condições para o EGP, além do ciclo de fervura $(4 \mathrm{~h}$ de fervura, $16 \mathrm{~h}$ a $20 \mathrm{~h}$ de secagem à $60^{\circ} \mathrm{C}+3^{\circ} \mathrm{C}, 4 \mathrm{~h}$ de fervura e $1 \mathrm{~h}$ de resfriamento em água a $20^{\circ} \mathrm{C}+3{ }^{\circ} \mathrm{C}$ ).

Os resultados foram confrontados com os requisitos mínimos relativos ao $5^{\circ}$ percentil inferior, estabelecidos na norma técnica EN 13353 (2008). Após os ensaios de cisalhamento, foram avaliadas as percentagens de falhas na madeira na superfície de ruptura da linha de cola. O delineamento estatístico utilizado foi $o$ inteiramente casualizado, com arranjo fatorial de $5 \times 2$, para cinco espécies e duas gramaturas, para cada tipo de adesivo (PVA e RF). 
TABELA 1. Delineamento experimental (espécie, adesivo e gramatura) utilizados.

\begin{tabular}{|c|c|c|}
\hline Espécie & Adesivo & Gramatura $\left(\mathrm{g} \mathrm{m}^{-2}\right)$ \\
\hline \multirow{2}{*}{ E. benthamii } & \multirow{2}{*}{ Poliacetato de vinila (PVAC) } & 150 \\
\hline & & 200 \\
\hline \multirow{2}{*}{ E. dunnii } & \multirow{2}{*}{ Poliacetato de vinila (PVAC) } & 150 \\
\hline & & 200 \\
\hline E. grandis & Poliacetato de vinila (PVAC) & 150 \\
\hline \multirow{2}{*}{ E. saligna } & \multirow{2}{*}{ Poliacetato de vinila (PVAC) } & 150 \\
\hline & & 200 \\
\hline \multirow{2}{*}{ E. urophylla } & \multirow{2}{*}{ Poliacetato de vinila (PVAC) } & 150 \\
\hline & & 200 \\
\hline \multirow{2}{*}{ E. dunnii } & \multirow{2}{*}{ Resorcina formaldeído (RF) } & 200 \\
\hline & & 300 \\
\hline \multirow{2}{*}{ E. grandis } & \multirow{2}{*}{ Resorcina formaldeído (RF) } & 200 \\
\hline & & 300 \\
\hline \multirow{2}{*}{ E. saligna } & \multirow{2}{*}{ Resorcina formaldeído (RF) } & 200 \\
\hline & & 300 \\
\hline \multirow{2}{*}{ E. urophylla } & \multirow{2}{*}{ Resorcina formaldeído (RF) } & 200 \\
\hline & & 300 \\
\hline
\end{tabular}

Os dados foram avaliados por meio de teste de Shapiro Wilk para normalidade, teste de Bartlett para homogeneidade das variâncias, e comparação de médias pelo teste de Tukey ao nível de probabilidade de 95\%. Os testes estatísticos foram realizados a partir do pacote estatístico Statgraphics XVII.

\section{Resultados}

\section{Propriedades físicas e químicas das madeiras de cinco espécies de Eucalyptus}

$\mathrm{Na}$ tabela 2 estão apresentados os valores médios de massa específica básica, anisotropia de contração, extrativos totais e pH, para as madeiras de cinco espécies de Eucalyptus. Os valores médios de massa específica básica para as espécies estudadas variaram de 0,39 para E. grandis e 0,53 para E. benthamii. Os valores médios de anisotropia de contração variaram de 2,14 para E. urophylla e 2,52 para E. benthamii.

Quanto às propriedades químicas, os valores médios de teor de extrativos totais variaram de 1,71\% para E. dunnii e 8,23\% para E. benthamii. Os valores médios de $\mathrm{pH}$ variaram de 4,19 para E. saligna e 4,77 para E. dunnii.

\section{Cisalhamento das juntas coladas com acetato de polivinila}

$\mathrm{Na}$ tabela 3 estão apresentados os resultados médios obtidos para os ensaios de cisalhamento das juntas coladas com acetato de polivinila, para os pré-tratamentos a seco, $24 \mathrm{~h}$ de imersão em água fria e fervura $6 \mathrm{~h}$. 
TABELA 2. Valores médios das propriedades físicas e químicas das madeiras estudadas.

\begin{tabular}{lcccc} 
Espécie & MEb & Ac & Extrativos totais (\%) & $\mathrm{pH}$ \\
\hline \multirow{2}{*}{ E. benthamii } & 0,531 & 2,52 & 8,23 & 4,34 \\
& $(6,14)$ & $(30,92)$ & $(16,50)$ & $(5,01)$ \\
\hline \multirow{2}{*}{ E. dunnii } & 0,488 & 2,40 & 1,71 & 4,77 \\
& $(11,23)$ & $(24,96)$ & $(25,48)$ & $(1,56)$ \\
\hline \multirow{2}{*}{ E. grandis } & 0,397 & 2,48 & 2,48 & 4,45 \\
& $(9,57)$ & $(21,92)$ & $(14,71)$ & $(2,47)$ \\
\hline \multirow{2}{*}{ E. saligna } & 0,472 & 2,18 & 2,47 & 4,19 \\
& $(11,87)$ & $(17,56)$ & $(13,47)$ & $(1,10)$ \\
\hline \multirow{2}{*}{ E. urophylla } & 0,524 & 2,14 & 4,74 & 4,22 \\
& $(13,24)$ & $(26,54)$ & $(8,33)$ & $(1,90)$ \\
\hline
\end{tabular}

MEb: massa específica básica; Ac: anisotropia de contração; valores entre parênteses indicam o coeficiente de variação.

TABELA 3. Valores médios da resistência ao cisalhamento das juntas coladas com acetato de polivinila (PVA D4) das espécies estudadas.

\begin{tabular}{|c|c|c|c|c|c|c|c|c|c|}
\hline \multirow[b]{2}{*}{ Tratamento } & \multicolumn{3}{|c|}{ Pré tratamento seco } & \multicolumn{3}{|c|}{ Pré tratamento 24 h água fría } & \multicolumn{3}{|c|}{ Pré tratamento fervura $6 \mathrm{~h}$} \\
\hline & $\begin{array}{c}\text { Cisalhamento } \\
\text { (MPa) }\end{array}$ & $\begin{array}{c}5^{\circ} \\
\text { Percentil } \\
\text { (MPa) }\end{array}$ & $\begin{array}{l}\text { Falha } \\
\text { (\%) }\end{array}$ & $\begin{array}{l}\text { Cisalhamento } \\
\text { (MPa) }\end{array}$ & $\begin{array}{c}5^{\circ} \\
\text { Percentil } \\
\text { (MPa) }\end{array}$ & $\begin{array}{l}\text { Falha } \\
\text { (\%) }\end{array}$ & $\begin{array}{l}\text { Cisalhamento } \\
\text { (MPa) }\end{array}$ & $\begin{array}{c}5^{\circ} \\
\text { Percentil } \\
\text { (MPa) }\end{array}$ & $\begin{array}{l}\text { Falha } \\
\text { (\%) }\end{array}$ \\
\hline E. benthamii 150 & $\begin{array}{c}10,69 \mathrm{AB} \\
(10,53)\end{array}$ & 9,06 & 90 & $\begin{array}{c}4,556 \mathrm{~A} \\
(22,55)\end{array}$ & 3,07 & 2 & $\begin{array}{c}2,66 \text { BCDE } \\
(33,91)\end{array}$ & 1,17 & $\mathrm{O}$ \\
\hline E. benthamii 200 & 11,09 A $(13,73)$ & 9,09 & 96 & $\begin{array}{l}4,65 A \\
(25,09)\end{array}$ & 2,86 & 1 & 3,68 A $(31,29)$ & 1,91 & 4 \\
\hline E. dunnii 150 & $\begin{array}{c}9,43 \mathrm{AB} \\
(17,97)\end{array}$ & 6,79 & 74 & 4,58 A $(15,77)$ & 3,62 & 1 & 1,88 E $(30,89)$ & 1,04 & 0 \\
\hline E. dunnii 200 & $10,17 A B(9,62)$ & 8,73 & 82 & 4,30 A $(21,92)$ & 3,36 & 1 & $2,11 \mathrm{DE}(29,18)$ & 1,25 & 0 \\
\hline E. grandis 150 & $\begin{array}{c}11,01 A B \\
(12,65)\end{array}$ & 8,77 & 86 & 4,72 A $(21,38)$ & 3,15 & 3 & $\begin{array}{c}2,32 \mathrm{BCDE} \\
(19,03)\end{array}$ & 1,67 & 0 \\
\hline E. grandis 200 & $\begin{array}{c}10,42 \mathrm{AB} \\
(12,99)\end{array}$ & 9,00 & 73 & 4,10 A $(19,02)$ & 3,16 & 3 & $\begin{array}{c}2,26 \mathrm{CDE} \\
(31,73)\end{array}$ & 1,56 & 0 \\
\hline E.saligna 150 & 9,21 B $(11,69)$ & 7,82 & 90 & 4,76 A $(23,16)$ & 3,19 & 2 & $\begin{array}{c}3,14 \mathrm{ABC} \\
(25,45)\end{array}$ & 1,78 & 2 \\
\hline E. saligna 200 & $\begin{array}{c}9,47 \mathrm{AB} \\
(13,14)\end{array}$ & 7,60 & 94 & 4,62 A $(16,08)$ & 3,83 & 3 & $\begin{array}{c}3,29 A B \\
(17,65)\end{array}$ & 2,95 & 2 \\
\hline E. urophylla 150 & $\begin{array}{c}10,69 \mathrm{AB} \\
(20,94)\end{array}$ & 7,37 & 63 & 4,52 A $(29,71)$ & 2,36 & 5 & $\begin{array}{c}3,11 \mathrm{ABC} \\
(28,68)\end{array}$ & 2,05 & 0 \\
\hline E. urophylla 200 & $\begin{array}{c}10,73 \mathrm{AB} \\
(15,11)\end{array}$ & 8,88 & 73 & $\begin{array}{l}4,46 \mathrm{~A} \\
(32,02)\end{array}$ & 2,52 & 2 & $\begin{array}{c}3,29 \text { ABCD } \\
(22,40)\end{array}$ & 2,19 & 0 \\
\hline
\end{tabular}

Médias seguidas de mesma letra na mesma coluna são estatisticamente iguais pelo teste de Tukey a 95\% de confiabilidade: valores entre parênteses referem-se ao coeficiente de variação em percentual. 
Os valores médios de cisalhamento no pré-tratamento a seco variaram de 9,21 MPa para juntas coladas de E. saligna com gramatura de $150 \mathrm{~g} \mathrm{~m}^{-2}$ a $11,09 \mathrm{MPa}$ para juntas coladas de E. benthamii com $200 \mathrm{~g} \mathrm{~m}^{-2}$. Foram constatadas diferenças estatisticamente significativas entre os tratamentos com diferentes espécies e gramaturas. As variações observadas no $5^{\circ}$ percentil inferior foi de 6,79 MPa para juntas coladas de E. dunnii com gramatura de 150 $\mathrm{g} \mathrm{m}^{-2}$ a 9,09 $\mathrm{MPa}$ para juntas coladas de E. benthamii com $200 \mathrm{~g} \mathrm{~m}^{-2}$. Com relação à percentagem de falhas na madeira, os valores médios variaram de $63 \%$ para juntas coladas de E. urophylla com gramatura de $150 \mathrm{~g} \mathrm{~m}^{-2}$ a $96 \%$ para juntas coladas de E. benthamii com $200 \mathrm{~g} \mathrm{~m}^{-2}$.

Para o pré-tratamento após $24 \mathrm{~h}$ de imersão em água fria, os valores médios de cisalhamento variaram de 4,10 MPa para juntas coladas de E. grandis com gramatura de 200 $\mathrm{g} \mathrm{m}^{-2}$ a 4,76 MPa para juntas coladas de E. saligna com 150 $\mathrm{g} \mathrm{m}^{-2}$. Os valores médios de $5^{\circ}$ percentil inferior variaram de 2,36 $\mathrm{MPa}$ para juntas coladas de E. urophylla com gramatura de $150 \mathrm{~g} \mathrm{~m}^{-2}$ a 3,83 $\mathrm{MPa}$ para juntas coladas de E. saligna com $200 \mathrm{~g} \mathrm{~m}^{-2}$. Os valores médios de percentagens de falhas na madeira ficaram abaixo de 5\% para todos os tratamentos.

Para o pré-tratamento com fervura de $6 \mathrm{~h}$, os valores médios de cisalhamento variaram de 1,88 MPa para juntas coladas de E. dunnii com gramatura de $150 \mathrm{~g} \mathrm{~m}^{-2}$ a 3,68 MPa para juntas coladas de E. benthamii com $200 \mathrm{~g} \mathrm{~m}^{-2}$. Os resultados de $5^{\circ}$ percentil inferior variaram de $1,04 \mathrm{MPa}$ para juntas coladas de E. dunnii com gramatura de $150 \mathrm{~g} \mathrm{~m}^{-2}$ a 2,95 MPa para juntas coladas de E. saligna com $200 \mathrm{~g} \mathrm{~m}^{-2}$. Com relação às falhas na madeira, a maioria dos tratamentos apresentou descolamento total da linha de cola, indicando que a fervura por período de $6 \mathrm{~h}$ é um procedimento muito prejudicial para madeiras coladas com adesivo PVA.

$\mathrm{Na}$ tabela 4 estão apresentados os efeitos da espécie e da gramatura na qualidade de colagem com adesivo PVA-D4.

TABELA 4. Efeito da espécie e da gramatura sobre a qualidade de colagem com adesivo PVA D4.

\begin{tabular}{|c|c|c|c|c|c|c|c|c|c|}
\hline \multirow[b]{2}{*}{ Fator principal } & \multicolumn{3}{|c|}{ Pré tratamento seco } & \multicolumn{3}{|c|}{ Pré tratamento 24 h água fria } & \multicolumn{3}{|c|}{ Pré tratamento fervura $6 \mathrm{~h}$} \\
\hline & $\begin{array}{l}\text { Cisalhamento } \\
\text { (MPa) }\end{array}$ & $\begin{array}{c}5^{\circ} \\
\text { Percentil } \\
\text { (MPa) }\end{array}$ & $\begin{array}{l}\text { Falha } \\
\text { (\%) }\end{array}$ & $\begin{array}{l}\text { Cisalhamento } \\
\text { (MPa) }\end{array}$ & $\begin{array}{c}5^{\circ} \\
\text { Percentil } \\
\text { (MPa) }\end{array}$ & $\begin{array}{l}\text { Falha } \\
(\%)\end{array}$ & $\begin{array}{l}\text { Cisalhamento } \\
\text { (MPa) }\end{array}$ & $\begin{array}{c}5^{\circ} \\
\text { Percentil } \\
\text { (MPa) }\end{array}$ & $\begin{array}{l}\text { Falha } \\
(\%)\end{array}$ \\
\hline \multicolumn{10}{|l|}{ Espécie } \\
\hline E. benthamii & 10,89 a $(12,19)$ & 8,70 & 93 & 4,61 a $(23,42)$ & 2,76 & 1 & 3,15 a $(29,66)$ & 1,32 & 2 \\
\hline E. dunnii & $\begin{array}{c}9,80 \mathrm{ab} \\
(14,36)\end{array}$ & 7,25 & 78 & 4,44 a $(18,82)$ & 3,32 & 1 & 1,98 b $(30,27)$ & 1,01 & 0 \\
\hline E. grandis & $\begin{array}{l}10,73 a \\
(10,80)\end{array}$ & 8,91 & 80 & 4,38 a $(21,16)$ & 3,04 & 3 & $2,29 \mathrm{~b}(25,47)$ & 1,60 & 0 \\
\hline E. saligna & 9,33 b $(12,20)$ & 7,75 & 92 & 4,69 a $(19,57)$ & 3,63 & 2 & 3,21 a $(18,53)$ & 2,16 & 1 \\
\hline E. urophylla & 10,71 a $(17,88)$ & 8,20 & 68 & 4,49 a $(30,26)$ & 2,12 & 3 & 2,94 a $(26,10)$ & 2,11 & 0 \\
\hline \multicolumn{10}{|l|}{ Gramatura } \\
\hline $150\left(\mathrm{~g} \mathrm{~m}^{-2}\right)$ & 10,18 a $(16,60)$ & 7,75 & 80 & 4,62 a $(22,30)$ & 2,79 & 2 & 2,62 a $(35,79)$ & 1,22 & 1 \\
\hline $200\left(\mathrm{~g} \mathrm{~m}^{-2}\right)$ & 10,40 a $(13,75)$ & 8,43 & 84 & 4,43 a $(23,23)$ & 2,99 & 2 & 2,82 a $(32,34)$ & 1,60 & 1 \\
\hline
\end{tabular}

Médias seguidas de mesma letra na mesma coluna são estatisticamente iguais pelo teste de Tukey a 95\% de confiabilidade: valores entre parênteses referem-se ao coeficiente de variação em percentual. 
Os resultados de cisalhamento entre as espécies de madeira de eucalipto variaram de 9,33 $\mathrm{MPa}$ (E. saligna) a 10,73 $\mathrm{MPa}$ (E. grandis) para o pré-tratamento a seco; de 4,38 MPa (E. grandis) a 4,69 $\mathrm{MPa}$ (E. saligna) para o pré-tratamento em água fria $24 \mathrm{~h}$; e, de 1,98 $\mathrm{MPa}$ (E. dunnii) a 3,21 $\mathrm{MPa}(E$. saligna) para o pré-tratamento em fervura de $6 \mathrm{~h}$. Para os tratamentos com diferentes gramaturas os valores médios de cisalhamento variaram de 2,62 $\mathrm{MPa}$ a 10,40 MPa para os três pré-tratamentos realizados.

Com exceção ao pré-tratamento com fervura $6 \mathrm{~h}$, todas as espécies e gramaturas apresentaram valores de $5^{\circ}$ percentil inferior acima do mínimo de 2,5 $\mathrm{MPa}$, exigido pela norma técnica EN 13353-2008. Para os pré-tratamentos com água fria $24 \mathrm{~h}$ e fervura $6 \mathrm{~h}$, foram obtidos baixos valores de percentagens de falhas na madeira.

\section{Cisalhamento das juntas coladas com resorcina-} formaldeído

$\mathrm{Na}$ tabela 5 estão apresentados os resultados médios obtidos para os ensaios de cisalhamento das juntas coladas com resorcina-formaldeído - RF, para os pré-tratamentos a seco, 24 h de imersão em água fria, fervura 6 horas e ciclo de fervura.

Os valores médios de cisalhamento no pré-tratamento a seco, variaram de 3,83 MPa para juntas coladas de E. grandis com gramatura de $200 \mathrm{~g} \mathrm{~m}^{-2}$ a 10,01 MPa para juntas coladas de E. grandis com $300 \mathrm{~g} \mathrm{~m}^{-2}$. Os resultados de $5^{\circ}$ percentil inferior variaram de $0,77 \mathrm{MPa}$ para juntas coladas de $E$. grandis com gramatura de $200 \mathrm{~g} \mathrm{~m}^{-2}$ a $6,46 \mathrm{MPa}$ para juntas coladas de E. dunnii com $300 \mathrm{~g} \mathrm{~m}^{-2}$. Com relação à percentagem de falhas na madeira, os valores variaram de $4 \%$ para juntas coladas de E. grandis com gramatura de $200 \mathrm{~g} \mathrm{~m}^{-2}$ a $53 \%$ para juntas coladas de E. dunnii com $300 \mathrm{~g} \mathrm{~m}^{-2}$.

TABELA 5. Valores médios da resistência ao cisalhamento das juntas coladas com resorcina-formaldeído RF.

\begin{tabular}{|c|c|c|c|c|c|c|c|c|c|c|c|c|}
\hline \multirow[t]{2}{*}{ Tratamento } & \multicolumn{3}{|c|}{ Pré tratamento seco } & \multicolumn{3}{|c|}{ Pré tratamento 24 h água fria } & \multicolumn{3}{|c|}{ Pré tratamento fervura $6 \mathrm{~h}$} & \multicolumn{3}{|c|}{ Pré tratamento ciclo } \\
\hline & $\begin{array}{l}\text { Cisalhamento } \\
\text { (MPa) }\end{array}$ & $\begin{array}{c}5^{\circ} \\
\text { Perc. } \\
\text { (MPa) }\end{array}$ & $\begin{array}{l}\text { Falha } \\
\text { (\%) }\end{array}$ & $\begin{array}{l}\text { Cisalhamento } \\
\text { (MPa) }\end{array}$ & $\begin{array}{c}5^{\circ} \\
\text { Perc. } \\
\text { (MPa) }\end{array}$ & $\begin{array}{l}\text { Falha } \\
(\%)\end{array}$ & $\begin{array}{l}\text { Cisalhamento } \\
\text { (MPa) }\end{array}$ & $\begin{array}{c}5^{\circ} \\
\text { Perc. } \\
\text { (MPa) }\end{array}$ & $\begin{array}{c}\text { Falha } \\
(\%)\end{array}$ & $\begin{array}{l}\text { Cisalhamento } \\
\text { (MPa) }\end{array}$ & $\begin{array}{c}5^{\circ} \\
\text { Perc. } \\
\text { (MPa) }\end{array}$ & $\begin{array}{l}\text { Falha } \\
(\%)\end{array}$ \\
\hline E. benthamii 200 & $\begin{array}{c}4,56 \mathrm{BC} \\
(34,56)\end{array}$ & 2,07 & 7 & $\begin{array}{c}1,78 \mathrm{C} \\
(34,28)\end{array}$ & 0,69 & 2 & $\begin{array}{l}3,01 \mathrm{Da} \\
(33,49)\end{array}$ & 1,22 & 3 & $\begin{array}{c}3,17 \mathrm{~B} \\
(37,77)\end{array}$ & 1,14 & 7 \\
\hline E. benthamii 300 & $\begin{array}{c}5,94 \mathrm{BC} \\
(33,95)\end{array}$ & 2,76 & 18 & $\begin{array}{c}3,71 \mathrm{ABC} \\
(33,08)\end{array}$ & 0,58 & 6 & $\begin{array}{c}4,36 \text { ABCD } \\
(33,51)\end{array}$ & 2,52 & 6 & $\begin{array}{c}3,39 \mathrm{~B} \\
(35,96)\end{array}$ & 1,19 & 4 \\
\hline E. dunnii 200 & $\begin{array}{l}7,84 \mathrm{AB} \\
(34,43)\end{array}$ & 3,43 & 39 & $\begin{array}{l}5,54 \mathrm{~A} \\
(18,46)\end{array}$ & 4,19 & 18 & $\begin{array}{c}5,96 \mathrm{AB} \\
(26,13)\end{array}$ & 2,67 & 38 & $\begin{array}{l}6,32 \mathrm{~A} \\
(14,51)\end{array}$ & 5,03 & 50 \\
\hline E. dunnii 300 & $\begin{array}{c}8,65 \mathrm{AB} \\
(17,42)\end{array}$ & 6,46 & 53 & $\begin{array}{l}6,36 \mathrm{~A} \\
(12,20)\end{array}$ & 5,17 & 50 & $\begin{array}{c}6,07 \mathrm{AB} \\
(21,88)\end{array}$ & 3,24 & 49 & $\begin{array}{l}5,16 \mathrm{AB} \\
(18,04)\end{array}$ & 3,92 & 56 \\
\hline E. grandis 200 & $\begin{array}{l}3,83 \mathrm{C} \\
(37,02)\end{array}$ & 0,77 & 4 & $\begin{array}{l}5,60 \mathrm{~A} \\
(35,51)\end{array}$ & 2,14 & 15 & $\begin{array}{l}6,42 \mathrm{~A} \\
(30,58)\end{array}$ & 3,11 & 1 & $\begin{array}{c}5,73 A B \\
(34,61)\end{array}$ & 2,11 & 6 \\
\hline E. grandis 300 & $\begin{array}{l}10,01 \text { A } \\
(27,96)\end{array}$ & 5,97 & 10 & $\begin{array}{l}5,38 \mathrm{~A} \\
(30,51)\end{array}$ & 2,57 & 6 & $\begin{array}{c}5,72 \mathrm{ABC} \\
(25,61)\end{array}$ & 2,03 & 13 & $\begin{array}{l}5,99 \mathrm{~A} \\
(30,21)\end{array}$ & 3,61 & 13 \\
\hline E. saligna 200 & $\begin{array}{c}4,74 \mathrm{BC} \\
(18,25)\end{array}$ & 1,76 & 10 & $\begin{array}{c}3,58 \mathrm{ABC} \\
(31,68)\end{array}$ & 2,62 & 7 & $\begin{array}{c}3,96 \mathrm{BCD} \\
(32,64)\end{array}$ & 2,07 & 3 & $\begin{array}{l}3,09 \mathrm{~B} \\
(35,61)\end{array}$ & 0,84 & 1 \\
\hline E. saligna 300 & $\begin{array}{c}5,52 \mathrm{BC} \\
(37,15)\end{array}$ & 2,30 & 4 & $\begin{array}{l}2,61 \mathrm{BC} \\
(39,39)\end{array}$ & 1,40 & 4 & $\begin{array}{l}2,96 \mathrm{D} \\
(30,10)\end{array}$ & 1,84 & 2 & $\begin{array}{l}4,52 \mathrm{AB} \\
(30,23)\end{array}$ & 1,61 & 7 \\
\hline E. urophylla 200 & $\begin{array}{c}5,20 \text { BC } \\
(38,25)\end{array}$ & 2,35 & 5 & $\begin{array}{l}5,09 A B \\
(32,60)\end{array}$ & 1,52 & 5 & $\begin{array}{c}4,90 \text { ABCD } \\
(37,07)\end{array}$ & 2,45 & 2 & $\begin{array}{c}5,56 \mathrm{AB} \\
(30,16)\end{array}$ & 2,85 & 8 \\
\hline E. urophylla 300 & $\begin{array}{l}5,56 \mathrm{BC} \\
a(34,55)\end{array}$ & 1,97 & 16 & $\begin{array}{c}3,45 A B C \\
(36,69)\end{array}$ & 1,02 & 11 & $\begin{array}{c}4,09 \text { ABCD a } \\
(32,96)\end{array}$ & 2,54 & 10 & $\begin{array}{c}3,76 \mathrm{AB} \\
(34,37)\end{array}$ & 1,52 & 5 \\
\hline
\end{tabular}

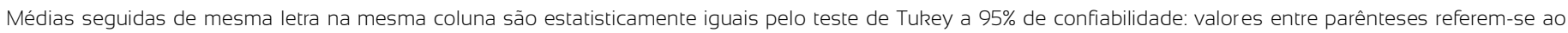
coeficiente de variação em percentual. 
Para o pré-tratamento após 24 h de imersão em água fria, os valores médios de cisalhamento variaram de 1,78 MPa para juntas coladas de E. benthamii com gramatura de $200 \mathrm{~g} \mathrm{~m}^{-2}$ a 6,36 MPa para juntas coladas de E. dunnii com $300 \mathrm{~g} \mathrm{~m}^{-2}$ Os resultados de $5^{\circ}$ percentil inferior variaram de $0,58 \mathrm{MPa}$ para juntas coladas de E. benthamii com gramatura de $300 \mathrm{~g} \mathrm{~m}^{-2}$ a 5,17 MPa para juntas coladas de E. dunnii com $300 \mathrm{~g} \mathrm{~m}^{-2}$. As percentagens de falhas na madeira variaram de $2 \%$ para juntas coladas de E. benthamii com gramatura de $200 \mathrm{~g} \mathrm{~m}^{-2}$ a $50 \%$ para juntas coladas de $E$. dunnii com $300 \mathrm{~g} \mathrm{~m}^{-2}$.

Para o pré-tratamento em fervura de $6 \mathrm{~h}$, os valores médios de cisalhamento variaram de 2,96 MPa para juntas coladas de E. saligna com gramatura de $300 \mathrm{~g} \mathrm{~m}^{-2}$ a $6,42 \mathrm{MPa}$ para juntas coladas de E. grandis com $200 \mathrm{~g} \mathrm{~m}^{-2}$. O $5^{\circ}$ percentil inferior após pré-tratamentos com água quente variaram de 1,22 MPa para juntas coladas de E. benthamii com gramatura de $200 \mathrm{~g} \mathrm{~m}^{-2}$ a 3,24 MPa para juntas coladas de E. dunnii com $300 \mathrm{~g} \mathrm{~m}^{-2}$. Com relação às falhas na madeira, a maioria dos tratamentos apresentou baixos percentuais, com exceção das juntas coladas de E. dunnii.

Para o pré-tratamento em ciclo de fervura, os valores médios de cisalhamento variaram de 3,09 $\mathrm{MPa}$ para juntas coladas de E. saligna com gramatura de $200 \mathrm{~g} \mathrm{~m}^{-2}$ a $6,32 \mathrm{MPa}$ para juntas coladas de E. dunnii com $200 \mathrm{~g} \mathrm{~m}^{-2}$. Os resultados do $5^{\circ}$ percentil inferior variaram de $0,84 \mathrm{MPa}$ para juntas coladas de E. saligna com gramatura de $200 \mathrm{~g} \mathrm{~m}-$ 2 a 5,03 MPa para juntas coladas de E. dunnii com $200 \mathrm{~g} \mathrm{~m}$ 2. Com relação às falhas na madeira, a maioria dos tratamentos apresentou baixos percentuais, com exceção das juntas coladas de E. dunnii que apresentaram valores intermediários.

$\mathrm{Na}$ tabela 6 estão apresentados os efeitos da espécie e da gramatura na qualidade de colagem com adesivo resorcina-formaldeído.

TABELA 6. Efeito da espécie e da gramatura sobre a qualidade de colagem com resorcina-formaldeído RF.

\begin{tabular}{|c|c|c|c|c|c|c|c|c|c|c|c|c|}
\hline & \multicolumn{3}{|c|}{ Pré tratamento seco } & \multicolumn{3}{|c|}{ Pré tratamento 24 h água fria } & \multicolumn{3}{|c|}{ Pré tratamento fervura $6 \mathrm{~h}$} & \multicolumn{3}{|c|}{ Pré tratamento ciclo } \\
\hline Fator principal & $\begin{array}{c}\text { Cisalhamento } \\
\text { (MPa) }\end{array}$ & $\begin{array}{c}5^{\circ} \\
\text { Perc. } \\
\text { (MPa) }\end{array}$ & $\begin{array}{l}\text { Falha } \\
(\%)\end{array}$ & $\begin{array}{l}\text { Cisalhamento } \\
\text { (MPa) }\end{array}$ & $\begin{array}{c}5^{\circ} \\
\text { Perc. } \\
\text { (MPa) }\end{array}$ & $\begin{array}{l}\text { Falha } \\
(\%)\end{array}$ & $\begin{array}{l}\text { Cisalhamento } \\
\text { (MPa) }\end{array}$ & $\begin{array}{c}5^{\circ} \\
\text { Perc. } \\
\text { (MPa) }\end{array}$ & $\begin{array}{l}\text { Falha } \\
\text { (\%) }\end{array}$ & $\begin{array}{c}\text { Cisalhamento } \\
\text { (MPa) }\end{array}$ & $\begin{array}{c}5^{\circ} \\
\text { Perc. } \\
\text { (MPa) }\end{array}$ & $\begin{array}{l}\text { Falha } \\
\text { (\%) }\end{array}$ \\
\hline \multicolumn{13}{|l|}{ Espécie } \\
\hline E. benthamii & $\begin{array}{l}5,39 \text { b } \\
(31,94)\end{array}$ & 2,51 & 14 & $\begin{array}{c}2,64 \mathrm{c} \\
(38,42)\end{array}$ & 1,48 & 4 & $\begin{array}{c}3,72 \text { b } \\
(40,42)\end{array}$ & 1,49 & 5 & $\begin{array}{l}3,30 \text { b } \\
(31,69)\end{array}$ & 1,15 & 5 \\
\hline E. dunnii & $\begin{array}{c}8,26 \text { a } \\
(25,26)\end{array}$ & 3,86 & 46 & $\begin{array}{l}5,90 \mathrm{a} \\
(16,92)\end{array}$ & 4,64 & 33 & $\begin{array}{l}6,01 \mathrm{a} \\
(27,11)\end{array}$ & 2,57 & 43 & $\begin{array}{l}5,79 a \\
(18,61)\end{array}$ & 4,10 & 53 \\
\hline E. grandis & $\begin{array}{l}6,77 \mathrm{ab} \\
(34,52)\end{array}$ & 0,88 & 7 & $\begin{array}{c}5,49 \text { a } \\
(22,09)\end{array}$ & 1,43 & 10 & $\begin{array}{l}6,05 \mathrm{a} \\
(32,78)\end{array}$ & 2,43 & 7 & $\begin{array}{c}5,87 \text { a } \\
(37,02)\end{array}$ & 2,82 & 9 \\
\hline E. saligna & $\begin{array}{c}5,10 \text { b } \\
(31,04)\end{array}$ & 1,74 & 7 & $\begin{array}{l}2,98 \text { bc } \\
(32,94)\end{array}$ & 1,64 & 5 & $\begin{array}{l}3,59 \text { b } \\
(44,37)\end{array}$ & 1,65 & 2 & $\begin{array}{c}3,71 \text { b } \\
(30,31)\end{array}$ & 0,89 & 4 \\
\hline E. urophylla & $\begin{array}{l}5,40 \mathrm{~b} \\
(39,45)\end{array}$ & 2,12 & 11 & $\begin{array}{l}4,51 \mathrm{ab} \\
(28,21)\end{array}$ & 1,20 & 8 & $\begin{array}{c}4,59 a b \\
(35,41)\end{array}$ & 2,23 & 6 & $\begin{array}{l}4,84 \mathrm{ab} \\
(37,00)\end{array}$ & 1,58 & 6 \\
\hline \multicolumn{13}{|l|}{ Gramatura } \\
\hline $200\left(\mathrm{~g} \mathrm{~m}^{-2}\right)$ & $\begin{array}{l}5,21 \mathrm{~b} \\
(49,12)\end{array}$ & 1,11 & 14 & $\begin{array}{c}4,43 a \\
(33,54)\end{array}$ & 1,06 & 7 & $\begin{array}{c}4,97 a \\
(43,69)\end{array}$ & 1,84 & 9 & $\begin{array}{l}4,96 a \\
(45,67)\end{array}$ & 1,04 & 17 \\
\hline $300\left(\mathrm{~g} \mathrm{~m}^{-2}\right)$ & $\begin{array}{c}7,23 a \\
(42,50)\end{array}$ & 2,28 & 20 & $\begin{array}{l}4,32 a \\
(30,21)\end{array}$ & 1,29 & 6 & $\begin{array}{l}4,90 a \\
(45,82)\end{array}$ & 1,74 & 18 & $\begin{array}{c}4,63 a \\
(39,99)\end{array}$ & 1,28 & 18 \\
\hline
\end{tabular}

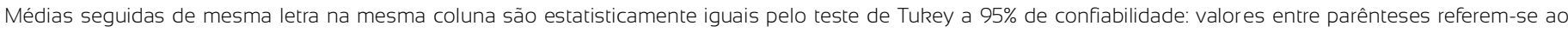
coeficiente de variação em percentual. 
Os resultados de cisalhamento entre as espécies variaram de 5,10 $\mathrm{MPa}$ (E. saligna) a 8,26 $\mathrm{MPa}$ (E. dunnii) para o prétratamento a seco; de 2,98 $\mathrm{MPa}$ (E. saligna) a 5,90 $\mathrm{MPa}$ (E. dunnii) para o pré-tratamento em água fria $24 \mathrm{~h}$; de 3,59 MPa (E. saligna) a 6,05 $\mathrm{MPa}$ (E. grandis) para o pré-tratamento em fervura 6 h; e, de 3,30 MPa (E. benthamii) a 5,87 MPa (E. grandis) para o pré-tratamento em ciclos de fervura. Para os tratamentos com diferentes gramaturas os valores médios de cisalhamento variaram de 4,32 $\mathrm{MPa}$ a 7,23 $\mathrm{MPa}$, para os quatro pré-tratamentos realizados.

Os valores médios de $5^{\circ}$ percentil inferior para as cinco espécies estudadas, variaram de $0,88 \mathrm{MPa}$ a 3,86 MPa para o pré-tratamento a seco; de 1,20 $\mathrm{MPa}$ a 4,64 $\mathrm{MPa}$ para o pré-tratamento em água fria $24 \mathrm{~h}$; de 1,49 $\mathrm{MPa}$ a 2,57 MPa para o pré-tratamento em fervura $6 \mathrm{~h}$; e, de $0,89 \mathrm{MPa}$ a 4,10 MPa para o pré-tratamento em ciclo de fervura. As percentagens de falhas na madeira foram baixas para todos os tratamentos, com exceção do E. dunnii.

Para os tratamentos com diferentes gramaturas os valores médios de cisalhamento variaram de 4,32 $\mathrm{MPa}$ a 7,23 MPa para os três pré-tratamentos realizados.

\section{DISCUSSÃO}

\section{Propriedades físicas e químicas da madeira de cinco espécies de Eucalyptus}

Os resultados médios de massa específica básica, obtidos para as cinco espécies de Eucalyptus, podem ser considerados como madeiras de média a baixa massa específica, e estão dentro da faixa de valores mencionados por Lorenzi (2002) para algumas espécies de eucalipto, como: E. grandis $\left(0,478 \mathrm{~g} \mathrm{~cm}^{-3}\right)$, E. urograndis $\left(0,502 \mathrm{~g} \mathrm{~cm}^{-3}\right)$ e E. saligna $\left(0,462 \mathrm{~g} \mathrm{~cm}^{-3}\right)$.

Cabe ressaltar que, as espécies mais empregadas na produção de painéis EGP no Brasil são as que possuem madeiras de baixa e média massa específica e, por conseguinte, maior porosidade e facilidade para penetração de adesivos de alta viscosidade, tais como: Tectona grandis $\left(0,480 \mathrm{~g} \mathrm{~cm}^{-3}\right.$ a $\left.0,640 \mathrm{~g} \mathrm{~cm}^{-3}\right)$, Pinus taeda $\left(0,320 \mathrm{~g} \mathrm{~cm}^{-3}\right.$ a 0,340 $\left.\mathrm{g} \mathrm{cm}^{-3}\right)$ e Eucalyptus grandis $\left(0,390 \mathrm{~g} \mathrm{~cm}^{-3}\right.$ a $\left.0,501 \mathrm{~g} \mathrm{~cm}^{-3}\right)$ (Lobão et al., 2011).
Para as madeiras avaliadas neste estudo, Klitzke (2007), sugeriu que, elas podem ser classificadas como de média a alta anisotropia, com valores entre 2,1 a 2,5. De maneira geral, as variações dimensionais e alta anisotropia são características indesejáveis a madeira, limitando o seu uso para diversas finalidades e exigindo técnicas adequadas de processamento e utilização (Keinert Jr et al., 1992). Entretanto, por meio de colagem de peças de madeira distribuídas aleatoriamente, como no caso de painéis EGP e madeira laminada colada, podem se minimizar os efeitos negativos da alta anisotropia de determinadas espécies.

Os resultados médios obtidos para os extrativos totais estão de acordo com as referências apresentadas na literatura, onde Fengel y Wegener (2003) citaram a faixa de $1 \%$ a 5\% para madeira de folhosas, e Sjöström (1981) relatou que o teor de extrativos totais é normalmente inferior a $10 \%$. Elevados teores de extrativos na madeira contribuem para contaminação de sua superfície, podem alterar o $\mathrm{pH}$, influenciando negativamente o processo de colagem por meio da pré-cura, e, ainda, podem gerar umectação e fluidez insuficientes.

Os valores médios de $\mathrm{pH}$ obtidos neste estudo, estão de acordo com aqueles mencionados por Stamm (1964), situados na faixa de 3,0 e 5,5. Johns y Niazi (1980) afirmam que a acidez da madeira depende da quantidade e dos tipos dos extrativos, e, normalmente, o $\mathrm{pH}$ do alburno situa-se entre 5 e 6 para quase todas as espécies e o pH do cerne entre 3 a 4.

\section{Cisalhamento das juntas coladas com acetato de polivinila}

Os resultados de resistência ao cisalhamento no prétratamento a seco, obtidos para as cinco espécies de Eucalyptus, foram satisfatórios em comparação aos apresentados na literatura, para madeiras de eucalipto, pinus e algumas espécies de madeiras tropicais, coladas com adesivo PVA. Iwakiri et al. (2013) encontraram para madeiras de Eucalyptus benthamii, valor médio de cisalhamento de 9,04 MPa. Endo, Trianoski e Iwakiri. (2017) obtiveram para Pinus taeda, valores entre 5,12 MPa e 
5,55 MPa. Já, Bila, Iwakiri, Trianoski e Prata (2016) encontraram para cinco espécies de madeiras tropicais (Eschweilera coriacea, Manilkara amazônica, Protium puncticulatum, Inga paraensis e Byrsonima crispa) valores entre 2,24 MPa e 8,45 MPa. Todos os tratamentos atenderam ao requisito mínimo de 2,5 $\mathrm{MPa}$, conforme estabelecido pela norma técnica EN 13353-2008. Quanto a percentagem de falhas na madeira, os valores obtidos atendem ao requisito mínimo de 40\% estabelecido pela norma técnica EN 13353-2008. As variações observadas neste estudo não foram tão amplas quanto as constatadas por Plaster, Oliveira, Abrahão e Braz (2008), os quais encontraram, para juntas coladas de madeiras de Eucalyptus spp com adesivo PVA, percentagens entre $26 \%$ a $91 \%$.

De acordo com a análise fatorial, as espécies $E$. benthamii, E. dunnii, E. grandis e E. urophylla apresentaram valores médios de cisalhamento estatisticamente iguais entre si, e superiores em comparação a E. saligna. Não foram constatadas diferenças significativas para as gramaturas de $150 \mathrm{~g} \mathrm{~m}^{-2}$ e $200 \mathrm{~g} \mathrm{~m}^{-2}$. Todos os tratamentos atenderam aos requisitos relativos ao $5^{\circ}$ percentil inferior e percentagem de falhas na madeira.

Os resultados de resistência ao cisalhamento no prétratamento por $24 \mathrm{~h}$ em água fria, obtidos para as cinco espécies de Eucalyptus, foram satisfatórios em comparação aos apresentados na literatura para madeiras de pinus e algumas espécies tropicais, coladas com adesivo PVA. Endo et al. (2017) encontraram para Pinus taeda valores de 3,36 MPa, e Iwakiri et al. (2016) obtiveram para madeiras de Protium puncticulatum e Dinizia excelsa, valores de 5,01 MPa e 5,07 MPa, respectivamente.

Não foram constatadas diferenças estatisticamente significativas entre os tratamentos com diferentes espécies e gramaturas. Com exceção do E. urophylla, todos as demais espécies e as duas gramaturas atenderam ao requisito mínimo de 2,5 $\mathrm{MPa}$ estabelecido pela norma técnica EN 13353-2008. Nas comparações com o pré-tratamento a seco, os resultados indicaram efeitos significativos da imersão em água fria na redução dos valores de cisalhamento da linha de cola e percentagem de falhas na madeira. Estudos realizados por Iwakiri et al. (2015) também revelaram baixos percentuais de falhas na madeira para as espécies Inga alba e Swartzia recurva, com valores de $2,0 \%$ e 3,0 \%, respectivamente, para o teste úmido, evidenciando a influência da umidade no cisalhamento da linha de cola.

Os resultados de resistência ao cisalhamento no prétratamento por $6 \mathrm{~h}$ em água quente, indicaram diferenças significativas entre os tratamentos. Para este tratamento não há referências bibliográficas para efeitos comparativos, tendo em vista que a colagem com adesivo PVA visa aplicações internas que não requerem alta resistência à umidade.

As espécies E. benthamii, E. saligna e E. urophylla apresentaram valores médios de cisalhamento estatisticamente iguais entre si, e superiores em relação às espécies E, dunni e E. grandis. Nenhuma espécie atendeu ao requisito mínimo referente ao $5^{\circ}$ percentil inferior de 2,5 MPa. Devido ao tratamento agressivo em água quente, constatou-se para todas as espécies e gramaturas, desolamento da linha de cola.

Para todos os pré-tratamentos realizados, as diferenças em massa específica básica, teor de extrativos totais e $\mathrm{pH}$ da madeira das cinco espécies de eucalipto, não afetaram significativamente os resultados de cisalhamento da linha de cola com adesivo PVA. Não foram constatadas também, diferenças significativas dos efeitos da gramatura de $150 \mathrm{~g}$ $\mathrm{m}^{-2}$ e $200 \mathrm{~g} \mathrm{~m}^{-2}$ na resistência ao cisalhamento das juntas coladas nos três pré-tratamentos efetuados. Trata-se de indicativo da melhor relação custo-benefício, com a possibilidade de aplicação de menor gramatura na colagem das madeiras destas espécies com adesivo PVA.

\section{Cisalhamento das juntas coladas com resorcina- formaldeído}

Os valores médios de cisalhamento no pré-tratamento a seco indicaram diferenças estatisticamente significativas entre as juntas coladas com diferentes espécies e gramaturas. O Eucalytpus dunnii e E. grandis apresentaram médias estatisticamente iguais entre si, e superiores em relação ao E. benrbamii, E. saligna e E. urophylla. Apenas o E. 
benthamii e E. dunnii atenderam ao requisito mínimo para $5^{\circ}$ percentil inferior de 2,5 MPa. Com relação à gramatura, o aumento de $200 \mathrm{~g} \mathrm{~m}^{-2}$ para $300 \mathrm{~g} \mathrm{~m}^{-2}$, resultou em maior valor médio de cisalhamento.

Para o pré-tratamento com $24 \mathrm{~h}$ de imersão em água fria, os E. dunnii e E. grandis apresentaram valores médios de cisalhamento estatisticamente superiores em relação às demais espécies. O E. dunnii foi a única espécie que atendeu ao requisito mínimo para $5^{\circ}$ percentil inferior de 2,5 $\mathrm{MPa}$, sendo a espécie que apresentou maior percentagem de falhas na madeira entre as cinco espécies estudadas. Não foram constatadas diferenças significativas para efeitos de gramatura.

Para os pré-tratamentos com fervura $6 \mathrm{~h}$ e ciclo de fervura, foram constatadas também as mesmas tendências encontradas para o pré-tratamento em água fria $24 \mathrm{~h}$. O E. dunnii e o E. grandis apresentaram valores médios de cisalhamento estatisticamente superiores em relação às demais espécies.

Para todos os pré-tratamentos realizados, o E. dunnii foi a espécie que apresentou maior percentagem de falhas na madeira, e, foi também a única espécie que atendeu ao requisito mínimo para $5^{\circ}$ percentil inferior de $2,5 \mathrm{MPa}$. A maior percentagem de falhas na madeira de E. dunnï pode ser atribuído à maior resistência da linha de cola ao cisalhamento, resultando em maior ruptura nas fibras de madeira.

Quanto à análise fatorial sobre os efeitos da gramatura, com exceção ao pré-tratamento a seco, para todos os demais pré-tratamentos, o aumento na gramatura de $200 \mathrm{~g}$ $\mathrm{m}^{-2}$ para $300 \mathrm{~g} \mathrm{~m}^{-2}$ não resultou em maiores valores de cisalhamento das juntas coladas.

Existem poucas referências na literatura sobre resistência ao cisalhamento de juntas coladas com adesivo resorcina-formaldeído, sendo que as disponíveis se referem aos resultados de ensaios após pré-tratamento a seco. Estudo realizado por Lima, Mori, Mendes, Trugilho e Mori (2008) para juntas coladas de clones de Eucalyptus com 250 $\mathrm{g} \mathrm{m}^{-2}$ de resorcina-formaldeído, revelou valores de tensões de cisalhamento entre 7,55 $\mathrm{MPa}$ a 9,07 $\mathrm{MPa}$ e falhas na madeira de 29 \% a 64\%. Já, Iwakiri et al. (2014) encontraram para madeiras de Tectona grandis, coladas com $350 \mathrm{~g} \mathrm{~m}^{-2} \mathrm{e}$ $450 \mathrm{~g} \mathrm{~m}^{-2}$ de resorcina-formaldeído, tensões de cisalhamento de 12,02 MPa a 14,17 MPa e falhas na madeira de $38,75 \%$ a $56,25 \%$.

Os resultados obtidos neste estudo foram um pouco inferiores ao relatados na literatura, porém cabe mencionar que a gramatura empregada de $200 \mathrm{~g} \mathrm{~m}^{-2}$ e $300 \mathrm{~g} \mathrm{~m}^{-2}$ foi menor em relação à utilizada por outros autores. Lima et al. (2008) relataram que vários fatores relacionados às madeiras das diferentes espécies de eucaliptos podem afetar a colagem, tais como: $\mathrm{pH}$, capacidade-tampão e tipos de extrativos, penetração do adesivo, viscosidade e teor de sólidos do adesivo, tempo e pressão durante a prensagem.

Nas comparações entre as espécies pode se destacar o desempenho do E. dunnii para todos os prétratamentos realizados. Analisando as propriedades físicas e químicas das madeiras das espécies estudadas, o E. dunnii foi a espécie que apresentou um baixo teor de extrativos totais, podendo ser a provável causa para obtenção de melhores resultados de colagem.

\section{CONCLUSÕES}

As madeiras de cinco espécies de Eucalyptus avaliadas neste estudo apresentaram densidade básica de média a baixa, e média a alta anisotropia; o teor de extrativos e pH estão dentro da faixa de valores referenciados na literatura; o $E$. dunnii foi a espécie que apresentou menor teor de extrativos totais.

Com base nos requisitos da norma técnica EN 153532008 para $5^{\circ}$ percentil inferior, as espécies E. benthamii, E. dunnii, E. grandis e E. saligna, apresentam um potencial interessante para produção de painéis EGP com adesivo PVA, para uso em ambiente interno e externo com cobertura.

Para as juntas coladas com adesivo resorcinaformaldeído, o E. dunniii foi a espécie que atendeu ao requisito mínimo da norma técnica EN 13353-2008 para $5^{\circ}$ percentil inferior, para todos os pré-tratamentos realizados. O baixo teor de extrativos totais da madeira desta espécie, pode ter contribuído para seu melhor desempenho na colagem. Portanto, pode se afirmar que, o E. dunnii possui 
grande potencial para a produção de madeira laminada colada, visando aplicações estruturais, inclusive, em ambientes externos.

Tanto para o PVA, quanto para a resorcinaformaldeído, o aumento da gramatura não melhorou de forma significativa a resistência ao cisalhamento das juntas coladas. Este resultado tem impacto positivo na relação custo-benefício, pois uma menor gramatura poderia ser utilizada na produção de painéis colados lateralmente e madeira laminada colada.

\section{REFERÊNCIAS}

Bila, N. F., Iwakiri, S., Trianoski, R., \& Prata, J. G. (2016). Avaliação da qualidade de juntas coladas de seis espécies de madeiras tropicais da Amazônia. Floresta, 46(4), 455-464. doi: $10.5380 /$ rf.v46i4.36311

European Standards. (2008). EN 13353. Solid wood panels (SWP) Requirements. Brussels: ES.

European Standards. (2008). EN 13354. Solid wood panels (SWP) - Bonding quality. Brussels: ES.

Comissão Panamericana de Normas Técnicas. (1972a). COPANT 461. Maderas: determinación del peso específico aparente. La Paz:

Comissão Panamericana de Normas Técnicas. (1972b). COPANT 459. Maderas: determinación del retratibilidad. La Pą:

Danawade, B. A., Malari, R. R., Patil, B. S., \& Hanamapure, R. S. (2014). Effect of finger joint on flexural strength of teak wood. International Journal of Engineering and Technology, 5(6), 4929-4937.

Endo, C., Trianoski, R., \& Iwakiri, S. (2017). Produção de EGP com diferentes adesivos PVAc e sistemas de prensagem. Floresta e Ambiente, 24,1-8. doi: 10.1590/2179-8087.147415

Fengel, D. \& Wegener, G. (2003). Wood chemistry, ultrastructure, reactions. Berlin/New York: Walter de Gruyter.

Fonte, A. P. N. \& Trianoski, R. (2015). Efeito da gramatura sobre a qualidade de colagem lateral da madeira de Tectona grandis. Revista de Ciências Agroveterinárias, 4(3), 224-233. doi: $10.5965 / 223811711432015224$

Iwakiri, S. (2005). Painéis de Madeira Reconstituida. Curitiba: FUPEF.

Iwakiri, S., Trianoski, R., Cunha, A. B., Prata, J. G., Hara, M., Bila, N. F., Luis, R. C. G., Araujo, R. D., \& Villas Bôas, B. T. (2013). Avaliação da resistência de juntas coladas da madeira de Eucalyptus benthamii com diferentes adesivos e faces de colagem. Scientia Forestalis, 41(99), 411-416.
Iwakiri, S., Matos, J. L. M., Trianoski, R., Parchen, C. F. A., Castro, V. G., \& Iwakiri, V. T. (2014). Características de vigas laminadas coladas confeccionadas com madeira de teca (Tectona grandis). Floresta e Ambiente, 21(2), 269-275. doi:10.4322/floram.2014.036

Iwakiri S., Trianoski, R., Nascimento, C. C., Gumane, C., Lengowski, E. C., Schardosin, F. A. \& Azambuja, R. (2015). Resistência das juntas coladas de madeiras de Inga alba (SW) Wild e Swartria recurva Poep. Cerne, 21(3), 457-463. doi:10.1590/01047760201521031844

Iwakiri, S., Trianoski, R., Fonte, A. P. N., França, M. C., Lau, P. C. \& Molleken, R. (2016). Potencial de uso de madeiras de Diniz̧ia excelsa Ducke e Protium puncticulatum J. F. Mach para produção de painéis EGP. Scientia Forestalis, 44(111), 709-717. doi: $10.18671 /$ scifor.v44n111.17

Johns, W. \& Niazi, K. (1980). Effect of pH and buffering capacity of wood and the gelation time of urea-formaldehyde resin. Wood and Fiber Science, 12, 255-263.

Scanavaca Jr, L. \& Garcia, J. N. (2004). Determinação das propriedades físicas e mecânicas da madeira de Eucalyptus urophylla. Scientia Forestalis, 65, 120-129.

Keinert Jr, S., Rozas, E. C. E., Esturion, J. A., Matsunaga, D. K., Michaque, M. A. M., \& Rincoski, C. R. (1992). Relação entre a contração e o teor de umidade da madeira de Pinus taeda e Pinus elliottii, em vários ângulos de grã. Ciência Florestal, 2(1), 81-86.

Klitzke, R. J. (2007). Secagem da madeira - Tecnologias aplicadas ao setor florestal brasileiro (pp. 271-341). Jerônimo Monteiro: Suprema Gráfica e Editora.

Lima, K. P. L., Mori, F. A., Mendes, L. M., Trugilho, P. F., \& Mori, C. L. S. O. (2008). Colagem da madeira de clones de Eucalyptus com três adesivos comerciais. Scientia Forestalis, 36(77), 73-77.

Lobão, M. S., Castro, V. R., Rangel, A., Sarto, C.; Tomazello Filho, M., Silva Junior, F. G., Camargo Neto, L., \& Bermudez, M. A. R. C. (2011). Agrupamento de espécies florestais por análises univariadas e multivariadas das características anatômica, física e química das suas madeiras. Scientia Forestalis, 18(1), 16-29.

Lopes, M. C., Muniz, G. I. B., Matos, J. L. M., Tanobe, V. O. A., Chinasso, C. A. F., \& Rosso, S. (2013). Resistência da linha de cola de painéis de Pinus taeda colados lateralmente com diferentes adesivos. Cerne, 19(4), 613-619.

Lorenzi, H. (2002). Árvores brasileiras: manual de identificação e cultivo de plantas arbóreas nativas do Brasil. Nova Odessa: Instituto Plantarum.

Marra, A. A. (1992). Technology of wood bonding: Principles in practice. New York: Van Nostrand Reinhold.

Martins, S. A. (2011). Adequação tecnológica da madeira de Eucalyptus benthamii Maiden et Cambridge para a produção de painéis colados lateralmente 
(PCL). Dissertação (Mestrado em Ciências Florestais), Universidade de Brasília. Brasília.

Pizzi, A. (1983). Wood adhesives: chemistry and technology. New York: Marcel Dekker.

Plaster, O. B., Oliveira, J. T. S., Abrahão, C. P., \& Braz, R. L. (2008). Comportamento de juntas coladas da madeira serrada de Eucalyptus sp. Cerne, 14(3), 251-258.

Sjöstrom, E. (1981). Wood chemistry. New York: Academic Press.

Stamm, A. J. (1964). Wood and cellulose science. New York: The Ronald Press Company.

Technical Association of the Pulp and Paper Industry. (2002a). TAPPI 204. Solvent extractives of wood and pulp. Atlanta.

Technical Association of the Pulp and Paper Industry. (2002b). TAPPI 252. $p H$ and Electrical Conductivity of Hot W ater Extracts of Pulp, Paper and Paperboard. Atlanta.

Tienne, D. L. C., Nascimento, A. M., Garcia, R. A., \& Silva, D. B. (2011). Qualidade de adesão de juntas de madeira de Pinus coladas em condições simuladas de serviço interna e externa. Floresta e Ambiente, 18(1), 16-29. doi: 10.4322/floram.2011.0191
Manuscrito recebido em 24 de outubro de 2019

Aceito em 23 de janeiro de 2020

Publicado em 2 de dezembro de 2020

Este documento deve ser citado como:

Trianoski, R., Iwakiri, S., \& Bonduelle, G. M. (2020). Qualidade das juntas coladas de madeira de cinco espécies de Eucalyptus com adesivos acetato de polivinila e resorcinaformaldeído. Madera y Bosques, 26(3), e2632064. doi: 10.21829/myb.2020.2632064

Madera y Bosques pelo Instituto de Ecología, A.C. é distribuída sob uma Licença Internacional Creative Commons Atribuição-NãoComercial ShareAlike 4.0. 